PSICOLOGÍA

IBEROAMERICANA
Psicología Iberoamericana ISSN: 1405-0943

revista.psicologia@ibero.mx

Universidad Iberoamericana, Ciudad de México México

\title{
Clínicas familiares: evaluando el efecto biopsicosocial de la pandemia (covid-19) en una comunidad urbana
}

Bazán Riverón, Georgina Eugenia; Osorio Guzmán, Maricela; Prado Romero, Carlos; Rodríguez Martínez, Jorge Iván; Ocampo-Jasso, José Antonio

Clínicas familiares: evaluando el efecto biopsicosocial de la pandemia (covid-19) en una comunidad urbana

Psicología Iberoamericana, vol. 29, núm. 3, Esp., e293330, 2021

Universidad Iberoamericana, Ciudad de México, México

Disponible en: https://www.redalyc.org/articulo.oa?id=133968747002

DOI: https://doi.org/10.48102/pi.v29i3.330

\section{(c) $(1)$}

Esta obra está bajo una Licencia Creative Commons Atribución 4.0 Internacional. 
Psicología Iberoamericana, vol. 29, núm. 3, Esp., e293330, 2021

Universidad Iberoamericana, Ciudad de México, México

Recepción: 01 Febrero 2021 Aprobación: 03 Agosto 2021

DOI: https://doi.org/10.48102/ pi.v29i3.330

Redalyc: https://www.redalyc.org/ articulo.oa?id= 133968747002
Artículos

\section{Clínicas familiares: evaluando el efecto biopsicosocial de la pandemia (covid-19) en una comunidad urbana}

Family clinics: Assessing the biopsychosocial effects of the (COVID-19) pandemic in an urban community

Georgina Eugenia Bazán Riverón gebrmx@yahoo.com.mx Facultad de Estudios Superiores Iztacala UNAM, México

(D) https://orcid.org/0000-0001-6582-6370 Maricela Osorio Guzmán mar1814@yahoo.com Facultad de Estudios Superiores Iztacala UNAM, México https://orcid.org/http://orcid.org/0000-0001-7798-5301 Carlos Prado Romero carlos.prado@iztacala.unam.mx Facultad de Estudios Superiores Iztacala UNAM, México

(D) https://orcid.org/0000-0003-0809-0672 Jorge Iván Rodríguez Martínez ivanromx@yahoo.com Facultad de Estudios Superiores Iztacala UNAM, México

(D) https://orcid.org/0000-0003-1403-9646 José Antonio Ocampo-Jasso jocampojasso@gmail.com Instituto Mexicano del Seguro Social (IMSS), México https://orcid.org/0000-0002-1426-9815

Resumen: Desarrollar intervenciones de salud adecuadas durante la pandemia por COVID-19 ha exigido analizar su impacto. El objetivo del estudio fue describir el efecto biopsicosocial general y por género en una comunidad urbana del Estado de México para intervenir adecuadamente. Participaron 639 personas de una Clínica Familiar (67\% mujeres, $27 \%$ hombres y $6 \%$ hombres-homosexuales). Se incluyeron diferentes grupos etarios $(M=43 ; \mathrm{DT}=23 ; \mathrm{R}: 8-97)$, mayoritariamente adultos (74\%), de clase social media (90\%) y de grado académico medio-superior (68\%). El estudio fue transversal, exploratorio, con muestreo por conveniencia-secuencial. Se encuestó mediante google-form sincrónicamente por videollamada. El análisis cuantitativo muestra un impacto biopsicosocial de la pandemia negativo en la muestra (mayormente en mujeres), duplicándose del $10 \%$ al $20 \%$ quienes consideran peor su salud general. Las personas con bienestar emocional y sin síntomas depresivos se reducen (37\% y $27 \%$ respectivamente). La presencia de trastornos conductuales, adicciones y violencia se presentan aproximadamente en el $50 \%$. La adherencia a medidas preventivas fue alta (40\%-70\%) y mayor en mujeres. Se concluye sobre la intervención multidisciplinariacomunitaria mediante clínicas familiares, analizando factores protectores y de riesgo.

Palabras clave: COVID-19, clínica familiar, impacto psicológico, salud, comunidad.

Abstract: Developing appropriate health interventions during the COVID-19 pandemic has required analyzing their impact. The objective of the study was to describe the general biopsychosocial effect by studying gender in an urban community in the State of Mexico to generate timely strategies. Six hundred and thirty-nine people from a family clinic ( $67 \%$ were women and $27 \%$ were men [ $6 \%$ of the men were homosexuals]) participated in the study. Different age groups were included $(M=43 ; S D=23 ; R$ : 8-97). Participants were mostly adults (74\%), middle income (90\%) and middle to 
upper educated (68\%). The study was cross-sectional, exploratory and implemented sequential convenience sampling. Data was collected using google-forms and video calls. The quantitative analysis shows a negative biopsychosocial impact of the pandemic on the sample (mostly in women), doubling from $10 \%$ to $20 \%$ of those who consider their general health worse. People who showed good emotional well-being and no depressive symptoms were reduced ( $37 \%$ and $27 \%$ respectively). The presence of behavioural disorders, addictions and violence are present in approximately $50 \%$ of the sample. Adherence to preventive measures was high (40\%-70\%) and higher in women. The study concluded that a multidisciplinary-community intervention through family clinics, analyzing protective and risk factors was needed.

Keywords: COVID-19, family clinic, psychological impact, health, community.

\section{Introducción}

De acuerdo con los informes de la Organización Mundial de la Salud (Organización Mundial de la Salud [OMS], 2020) el 31 de diciembre de 2019, en Wuhan, China, se informó la presencia de múltiples casos de neumonía sin poder precisar su etiología. Días después se confirmó la causa, un nuevo coronavirus: el "SARS-CoV-2". Dicho virus se propagó rápidamente y provocó la llamada enfermedad por coronavirus 2019, la cual se extendió a cientos de países, ocasionando millones de casos de COVID-19, por lo que la OMS la catalogó como pandemia. Ésta debería ser controlada mediante la acción coordinada de todos los sectores gubernamentales, atendiendo aspectos de prevención, diagnóstico y tratamiento para enfrentarla efectivamente (BBC News, 2020a). El 30 de enero de 2020, la OMS declaró la situación como Emergencia de Salud Pública de Importancia Internacional (ESPI). El 11 de febrero de 2020, el Comité Internacional de Taxonomía de Virus (ICTV, por sus siglas en inglés: International Committee on Taxonomy of Viruses) señala que el nombre oficial del virus causante de la enfermedad es $S A R S-C o V-2$ (BBC News, 2020b).

En cuanto a su etiología y transmisión, se sugiere que el SARS-CoV-2 inicialmente habitó en un huésped animal y posteriormente migró a seres humanos (Andersen et al., 2020; OMS, 2020). Su transmisión, se da de persona a persona mediante tres principales mecanismos (AragónNogales et al., 2020; OMS, 2020): el primero, por contacto con gotas respiratorias expulsadas por boca y nariz de alguien infectado; el segundo, por contacto con superficies contaminadas y tocarse los ojos, nariz o boca; y el tercero, al inhalar partículas infectantes a manera de aerosol. El hecho de identificar estos mecanismos ha sido fundamental para desarrollar estrategias de prevención y control de la enfermedad. Lo que dio pie a planear y aplicar las medidas en las tres fases de vigilancia epidemiológica que se describen a continuación (Gobierno de la Ciudad de México [CDMX]) \& Secretaría de Salud, 2020, 2021).

La primera fase inició el 28 de febrero de 2020 con la vigilancia epidemiológica, cuando se confirman los primeros dos casos de COVID-19 en México. El 11 de marzo de 2020, la OMS calificó como pandemia la enfermedad denominada COVID-19, porque los casos 
confirmados superaron a los 118,000 en 114 países y el número de fallecidos ascendía a 4,291 (BBC News, 2020b).

La segunda fase de la pandemia comenzó el 24 de marzo con la dispersión comunitaria, mostrando cientos de contagios. Se asumió como caso sospechoso de COVID-19 a toda persona que en los últimos siete días hubiera presentado al menos dos síntomas clínicos, como tos, fiebre o cefalea. Así mismo, se consideraba si también estaba presente al menos uno de los siguientes síntomas: disnea (dato de gravedad), artralgias, mialgias, odinofagia, rinorrea, conjuntivitis o dolor torácico (Centers for Disease Control and Prevention [CDC], 2020; Tesini, 2020).

La tercera fase epidemiológica, comenzó el 21 de abril de 2020, se consolidó con la presencia de miles de contagios. Es a mediados del mes de marzo que el gobierno mexicano instaura el distanciamiento social, se suspenden las actividades que propicien acumulaciones de personas. La población de todas las edades es restringida a sus hogares, viéndose obligada a convivir todo el día con sus familias, teniendo que realizar todas las actividades cotidianas pero ahora dentro de sus casas.

Las personas se ven afectadas por las pérdidas experimentadas y la incertidumbre. Hechos que se generan en la vivencia de situaciones altamente estresantes como el distanciamiento social por la pandemias, la violencia, los conflictos bélicos y desastres naturales, entre otros. Se ha comprobado que derivado de estas vivencias las personas muestran mayor vulnerabilidad para presentar trastornos psicológicos (InterAgency Standing Committee [IASC], 2007; World Health Organization [WHO], 2004; Zaccarelli et al., 2006). Los informes mundiales recuperados de la Organización Panamericana de la Salud [OPS] (2012, 2016) establecen que la prevalencia de padecimientos psiquiátricos y psicológicos severos (psicosis, depresión severa y trastornos de ansiedad) en la población adulta se duplican al vivir un desastre, es decir, doce meses antes del evento los trastornos pueden presentarse en un 2\% a $3 \%$ de la población, mientras que doce meses después del evento se eleva hasta el $3 \%$ o 4\%. Lo mismo sucede con padecimientos leves de depresión y ansiedad, los cuales muestran una prevalencia de $10 \%$ doce meses antes del desastre, y entre $15 \%$ y $20 \%$ al evaluar doce meses después del evento.

Desde hace décadas se ha argumentado en múltiples comunicados sobre salud mental a nivel mundial por la OMS (WHO, 2004) y la OPS (2010, 2012), que ante eventos altamente estresantes (como puede ser una pandemia) se han detectado la aparición de trastornos como el temor, ansiedad, depresión, así como la exacerbación de problemas sociales por pérdidas financieras, violencia y adicciones.

Actualmente Galindo-Vázquez et al. (2020) aseveran que hay más personas con altos niveles de ansiedad y depresión que en otras pandemias. Así mismo autores como Taylor et al. (2008) consideran importante dar contexto a cómo viven las personas dicho evento, documentando que el distanciamiento social ocasiona sufrimiento psicológico. En revisiones actuales de la literatura (Brooks et al., 2020; Rajkumar, 2020) identifican efectos psicológicos negativos tras la pandemia como confusión, ira, estrés 
postraumático y la disminución en la satisfacción con la vida (Díaz et al., 2007; Hawryluck et al., 2004; Jeong et al., 2016; Li, Yang et al., 2020).

En este mismo sentido, a nivel organizacional se ha identificado el efecto de factores estresantes derivados de la cuarentena, tales como temor de infección, frustración, aburrimiento, suministros insuficientes, información inadecuada, pérdidas financieras y estigma que permanecieron aún después de la cuarentena (Lai et al., 2020; Li, Yang et al., 2020; Liu et al., 2020; McMullan et al., 2016). Otros autores han observado también como consecuencias del distanciamiento y la incertidumbre la aparición de ansiedad, depresión y trastornos del sueño en trabajadores de la salud (Huarcaya-Victoria, 2020; Robles et al., 2020) y en el personal académico (Saldívar-Garduño \& Ramírez-Gómez, 2020).

En dos estudios internacionales, uno con población hispana (LandaBlanco et al., 2020) y el otro a nivel mundial (Yuksel et al., 2021) se señala un aumento en los síntomas psicológicos (depresión, ansiedad y comportamientos dañinos) como respuesta a la pandemia. Además ellos observan aspectos psicosociales como predictores, identificando que ser mujer, joven y trabajar en el ámbito de salud, agrava el impacto de la pandemia. Así también se obtiene que los problemas familiares y financieros son la principal preocupación en la mayoría de los países estudiados, incluido México. Estas evidencias muestran la trascendencia de considerar variables psicosociales, en la salud general y psicológica, que permitan orientar mejor las intervenciones, adaptándolas a poblaciones que dadas sus características presenten mayor vulnerabilidad.

Específicamente, en investigaciones con población mexicana otros autores han identificado importantes afectaciones, considerando algunas variables psicosociales como género y estado civil, notando que las mujeres muestran mayor deterioro en la calidad de sueño, mas ansiedad y depresión (Terán-Pérez et al., 2021). Así mismo, otros estudios informan que ser mujer, joven, soltera y convivir con un rango de entre tres a cinco personas durante el distanciamiento social, fungen como factores de riesgo para mostrar más pensamientos intrusivos, evitación, estrés, ansiedad y depresión (Ramírez et al., 2020; Valdez et al., 2020).

Otras variables importantes relacionadas con el manejo de la actual pandemia tienen que ver con la adherencia a medidas preventivas. Saldívar-Garduño y Ramírez-Gómez (2020) a pesar de obtener en su muestra una adherencia relativamente satisfactoria, observan que entre $5 \%$ y $10 \%$ de las personas no cumplen ninguna de las recomendaciones sanitarias. Betancourt-Ocampo et al. (2021) identifican qué variables pueden predecir la adherencia, exploran algunas variables como género y edad en relación al riesgo percibido de contraer COVID-19. Ellos notan que son las mujeres quienes se adhieren más a las medidas de higiene viéndolas como más útiles- y presentando mayor preocupación respecto contraer COVID-19 a diferencia de los hombres.

Las aportaciones de los estudios anteriores se pueden vincular también con aspectos como el conocimiento sobre COVID-19, el sesgo negativo o positivo ante la probabilidad de contagio, la adherencia, la creación de normas subjetivas (influencia del entorno inmediato para la adherencia), 
lo cual ha sido poco investigado (Caldera-Villalobos et al., 2020). En México, donde la relación con las autoridades se ha caracterizado por la desconfianza, y dados los cambios sociopolíticos actuales, es por demás interesante indagar de qué manera media la credibilidad en las autoridades la adherencia a medidas preventivas.

Ante este panorama, es imprescindible obtener datos del efecto que la pandemia puede tener sobre las personas, de manera general y por género. Más aun, en México se requieren directrices claras para optimizar los recursos existentes, como las 27,689 Clínicas Familiares con que se cuenta en el país, de las cuales el $39.5 \%$ pertenecen al ámbito urbano, según el Segundo Informe de Trabajo de la Secretaria de Salud de México (Secretaria de Salud, 2020). En estas instancias se desarrolla el Modelo de Atención Primaria de la Salud Integral (APS-I Mx), que pretende favorecer el acceso universal de atención en todos los niveles, subrayando la atención multidisciplinaria en la prevención mediante la participación comunitaria.

En consecuencia, el objetivo de la presente investigación fue describir el efecto biopsicosocial general y por género de la pandemia en una comunidad urbana del Estado de México. La información permitirá la planeación de intervenciones multidisciplinarias mejor orientadas y oportunas para la salud de la comunidad.

\section{Método}

\section{Participantes}

El diseño de este estudio fue cuantitativo, transversal, exploratorio y descriptivo. La muestra se conformó por conveniencia y de manera secuencial por 639 personas pertenecientes a 89 familias con un promedio de 7.1 integrantes (D.T. $=4$ ). La edad promedio fue de 43 años (Rango: 8 -97; DT $=23$ ), el $74 \%$ adultos, $14 \%$ adolescentes, $11 \%$ adultos mayores, el $1 \%$ niños. Los participantes son pacientes del servicio de psicología en una Unidad de Medicina Familiar (UMF) del Instituto Mexicano del Seguro Social (IMSS); fueron contactados vía telefónica por medio de los datos de su expediente clínico. Se calculó el índice Hollingshead (1975), basado en dos indicadores (ocupación y grado académico), agrupando la variable en una escala de cinco puntos: la más baja: nivel I y la más alta: nivel V. El 90\% de muestra se encuentra entre el punto II y III y $10 \%$ en el punto IV. El criterio de inclusión, consideró derechohabientes del Servicio de Psicología de una UMF en el Estado de México. Quienes tuvieran al menos un dispositivo inteligente y desearan participar. El escenario fue virtual, mediante aplicación de mensajería instantánea y sala de videoconferencias sincrónicas [Zoom]. 


\section{Instrumento}

Se elaboró un cuestionario ad hoc de 40 ítems, diseñado y distribuido mediante la plataforma google forms para valorar el efecto del distanciamiento social. Los ítems preguntaron sobre el comportamiento regular un mes antes del distanciamiento y durante éste. Las dimensiones que conforman el instrumento de evaluación se decriben a continuación.

\section{Variables sociales}

Que comprendieron: a. Aspectos sociodemográficos (género, edad, tipo de familia, estado civil, nivel de estudios, ocupación, lugar de residencia, edad de las personas con quien vive, tipo de vivienda; b. Impacto social del distanciamiento (en actividad laboral/escolar, en convivencia social y familiar máximo número de días continuos sin salir); c. Presencia de violencia; d. Percepción del manejo gubernamental de la pandemia y e. Medio elegido para estar informado sobre COVID-19.

\section{Variables de salud general}

Considerando: a. Adherencia a las conductas de prevención y normas sociales de prevención, obteniendo información sobre la práctica personal y de su entorno inmediato; b. Conocimientos básicos sobre COVID-19; c. Medios de transmisión y medidas de prevención y d. Impacto en la salud física en general.

\section{Variables psicológicas}

Las cuales incluyeron: a. Impacto del distanciamiento social en el estado de ánimo general; b. Ansiedad; c. Depresión y d. Cambios conductuales (estilo de vida, comportamientos repetitivos y adicciones).

Se hicieron pruebas de validez de contenido, constructo y apariencia (concordancia entre expertos Kappa=1; IC.95\%: 0-1) mediante un panel multidisciplinario de seis expertos (de las áreas de medicina, enfermería, psicología); se realizó un breve estudio preliminar en una muestra de 60 diadas, para verificar claridad en la redacción (concordancia entre expertos Kappa=.85-.94; IC.95\%: 0.84-0.96), y homogeneidad en las respuestas. Obteniendo una nueva versión de 38 ítems (se eliminaron 2 ítems del área de conocimiento por no obtener datos satisfactorios en la dificultad, homogeneidad y por tanto en su poder discriminativo. Los datos sobre la presencia y severidad de síntomas de ansiedad y depresión se tomaron de su expediente clínico. En el caso de los adultos, han sido evaluados (previamente y durante la pandemia) mediante entrevista clínica y el Inventario de Depresión de Beck (de sus siglas en inglés -BDI-) validado para población mexicana (González et al., 2015) y el inventario de Ansiedad de Beck (de sus siglas en inglés -BAI-) validado en población mexicana (Robles et al., 2001). En el caso de niños y adolescentes se evaluó 
la depresión mediante datos en expediente obtenido con el instrumento de auto-informe CDI (del inglés, Children's Depression Inventory) versión realizada por Del Barrio y Carrasco (2004); y la ansiedad se obtuvo mediante los datos de expediente clínico que incluye entrevista clínica a padres y maestros, ya que los participantes son atendidos en el servicio psicológico de la unidad que mantiene contacto con los centros escolares. En el ítem que valora la violencia se incluyó el Violentómetro (Instituto Politécnico Nacional [IPN], 2009). Este Instrumento que fue diseñado por la Unidad Politécnica de Gestión con Perspectiva de Género (UPGPG). Es un material gráfico y didáctico que se divide en tres escalas o niveles de diferentes colores y a cada uno corresponde una situación de alerta.

\section{Procedimiento}

El presente estudio siguió los lineamentos éticos de la Asociación Americana de Psicología, así como lo estipulado en el Código Ético del Psicólogo de la Sociedad Mexicana de Psicología. Fue aprobado por la Comisión Mixta de la UMF del IMSS y del Proyecto de Enfermedades Crónicas, adscrito a la Unidad de Investigación Interdisciplinaria en Ciencias de la Salud y Educación.

Se contactó a los pacientes vía telefónica y una vez que aceptaron participar los mayores de edad firmaron el consentimiento informado y en el caso de tener menores de edad que desearan participar en la investigación, los adultos autorizaron ésta (mediante su supervisión), los menores dieron su asentimiento informado. En este primer contacto se enfatizó la invitación e inclusión de adolescentes y jóvenes (lo que permitió contar con apoyo en el manejo digital personalizado a distancia). Posteriormente se les incluyó en una aplicación de mensajería instantánea (grupo en whatsapp) en el cual se dieron instrucciones, videos de apoyo para habilitar el dispositivo inteligente y para ingresar a ligas de información, proporcionando asesoría personalizada. Una vez concluida esta fase se promovió la asistencia a una sesión de instrucción, la cual fue impartida mediante aplicaciones de mensajería instantánea y/o por video llamada (videoconferencia sincrónicas Zoom). La sesión constó de dos partes, una de sensibilización y otra de capacitación. La sensibilización subrayó la importancia de participar teniendo un escenario social virtual común en el grupo familiar. Se explicaron los beneficios de estrechar brechas intergeneracionales aprendiendo de los jóvenes de sus familias el manejo de las Tecnologías de la Información y la Comunicación (TIC) y la importancia de mantenerse conectados socialmente. La capacitación, se enfocó a la habilidad para ingresar a conferencias zoom, a mensajería instantánea y para responder y enviar instrumentos en google forms. Se programaron 12 sesiones, con horarios diversos, repitiendo la misma estructura descrita, donde las personas podían elegir cuando ingresar, pudiendo hacerlo las veces que fuera necesario para practicar los conocimientos adquiridos y resolver sus dudas. Se dieron por terminadas las sesiones de sensibilización y capacitación una vez que los participantes 
lograron responder y enviar un formulario de práctica en google form adecuadamente.

Para recoger la información de este estudio se envió mediante mensajería instantánea la liga de acceso al instrumento, la aplicación se llevó a cabo durante el distanciamiento social en los meses de abril y mayo. Se programaron diez sesiones con horarios diversos para participar sincrónicamente en video-llamadas donde se hicieron las aplicaciones dirigidas a modo de entrevista colectiva para grupos de hasta 80 personas.

\section{Análisis de resultados}

Se procesó la información de google form, exportando la base de datos al programa SPSS versión 25. Se realizó estadística descriptiva para la muestra en general, para las variables escalares se calcularon medidas de tendencia central y dispersión, para las variables ordinales y nominales se calcularon porcentajes. Se analizaron las diferencias de género mediante $\mathrm{Ji}^{2}$. Primero se compararon género femenino, masculino y homosexuales masculinos, antes y durante la pandemia. Debido a que no se encontraron diferencias significativas entre categorías de género antes de la pandemia, sólo se presentan los resultados después de la pandemia. Cabe señalar que en este último análisis las categorías hombres y hombres-homosexuales no mostraron diferencias en las variables consideradas, por lo que el estudio del género fue binario. Se tabuló la información.

\section{Resultados}

\section{Aspectos sociales}

\section{Sociodemográficos}

Se invitó a 697 pacientes, de los cuales aceptaron participar 639. Como se puede observar en la Tabla 1, la mayoría de los participantes fueron de género femenino (67\%). El grupo de edad predominante en la muestra se compuso por adultos (74\%). El tipo de familia mayoritario fue la familia extensa (36\%), seguido por la familia nuclear (32\%). Respecto al estado civil la mayoría informaron estar casados (47\%) o solteros (47\%). En relación con grado escolar el $37 \%$ poseen una licenciatura terminada y $31 \%$ bachillerato terminado. La ocupación con mayor porcentaje fue dedicarse a actividades profesionales o empresariales (31\%) y el ser estudiante (24\%). Al considerar el rango de edad de las personas con quien conviven los participantes, se observó mayormente el de 18-29 años (62\%), seguido por el de personas mayores de 60 años (47\%). Sobre los tipos de vivienda, los participantes refirieron cuatro (casa con patio o jardín -la más frecuente 59\%-, casa, departamento con vista exterior y departamento con balcón) (Tabla 1). 
Tabla 1

Tabla 1

Datos sociodemográficos de la muestra

\begin{tabular}{|c|c|c|c|c|c|}
\hline \multirow{2}{*}{$\begin{array}{l}\text { Categoría } \\
\mathrm{N}=639\end{array}$} & \multicolumn{5}{|l|}{ Porcentaje } \\
\hline & & & & & \\
\hline \multirow[t]{2}{*}{ Género } & Femenino & Masculino & No binario & & \\
\hline & 67 & 27 & 6 & & \\
\hline \multirow[t]{3}{*}{ Edad } & $<9$ años & Adolescente & Adultos & Adultos & \\
\hline & & & & Mayores & \\
\hline & 1 & 14 & 74 & 11 & \\
\hline Tipo de & Monoparental & Nuclear & No/sanguínea & Extensa & \\
\hline Familia & 12 & 32 & 20 & 36 & \\
\hline Estado & Casado & Soltero & Unión Libre & Viudo & \\
\hline civil & 47 & 47 & 3 & 3 & \\
\hline \multirow[t]{3}{*}{ Estudios } & Primaria & Básica & Bachillerato & Licenciatura & Posgrado \\
\hline & & media & & & \\
\hline & 11 & 18 & 31 & 37 & 3 \\
\hline \multirow[t]{3}{*}{ Ocupación } & Empresario/ & Estudia & Independiente & Jubilado & Operativo \\
\hline & profesional & & microempresa & & y oficios \\
\hline & 31 & 24 & 8 & 14 & 23 \\
\hline Edad & $<18$ años & 18-29 años & 30-49 años & $50-60$ años & $>60$ años \\
\hline grupo de & & 62 & & & \\
\hline convivencia & 34 & & 42 & 36 & 47 \\
\hline \multirow{3}{*}{$\begin{array}{l}\text { Tipo de } \\
\text { vivienda }\end{array}$} & Casa & Casa con & Depto. vista & Depto. con & \\
\hline & & patio & exterior & balcón & \\
\hline & 13 & 59 & 15 & 13 & \\
\hline
\end{tabular}

\section{Impacto del distanciamiento social}

En cuanto al impacto de la pandemia en el ámbito laboral (Tabla 2), se observaron cambios en la actividad de los participantes antes y después de la pandemia. Se puede notar que del $80 \%$ que salía de casa a trabajar antes la pandemia, $40 \%$ se tuvo que quedar en casa por despidos y restricciones por tener actividad no esencial. Mientras que sólo el 23\% pudo quedarse en casa y mantener relativamente estable su empleo y economía. En el 
caso de quienes sólo estaban en casa o tenían actividades mixtas (dentro y fuera), ahora con la pandemia se notó un aumento importante (19\% y $21 \%$, respectivamente), así mismo se observó que el $11 \%$ que no trabajaba se mantiene igual (jubilados y/o pensionados, amas de casa o dependientes de sus hijos). Sobre la convivencia social y familiar los datos obtenidos son muy similares la mayoría antes (74\%) tenían actividades dentro de casa y fuera el $26 \%$, sin embargo, a partir de la pandemia estos porcentajes se reducen; además el 15\% refiere que actualmente no tiene convivencia social y el $14 \%$ ha dejado de ver a sus familiares. Finalmente se realizó un análisis de las diferencias por género antes de la pandemia en las variables mencionadas sin encontrar diferencias significativas $\left(\mathrm{ji}^{2}=12.39-\mathrm{ji}^{2}=50.4\right.$; $\mathrm{p}>.05)$. A diferencia de después de la pandemia, encontrando diferencias significativas (Tabla 2) dado que más mujeres trabajan dentro de casa y más hombres fuera. Así mismo, la vida social ha cambiado, pues más mujeres socializan dentro de casa. Sobre la convivencia familiar también hay diferencias más mujeres que hombres la viven al interior de casa. 
Tabla 2

Tabla 2

Impacto del distanciamiento social en las áreas laboral, de convivencia social y familiar muestra general y por género

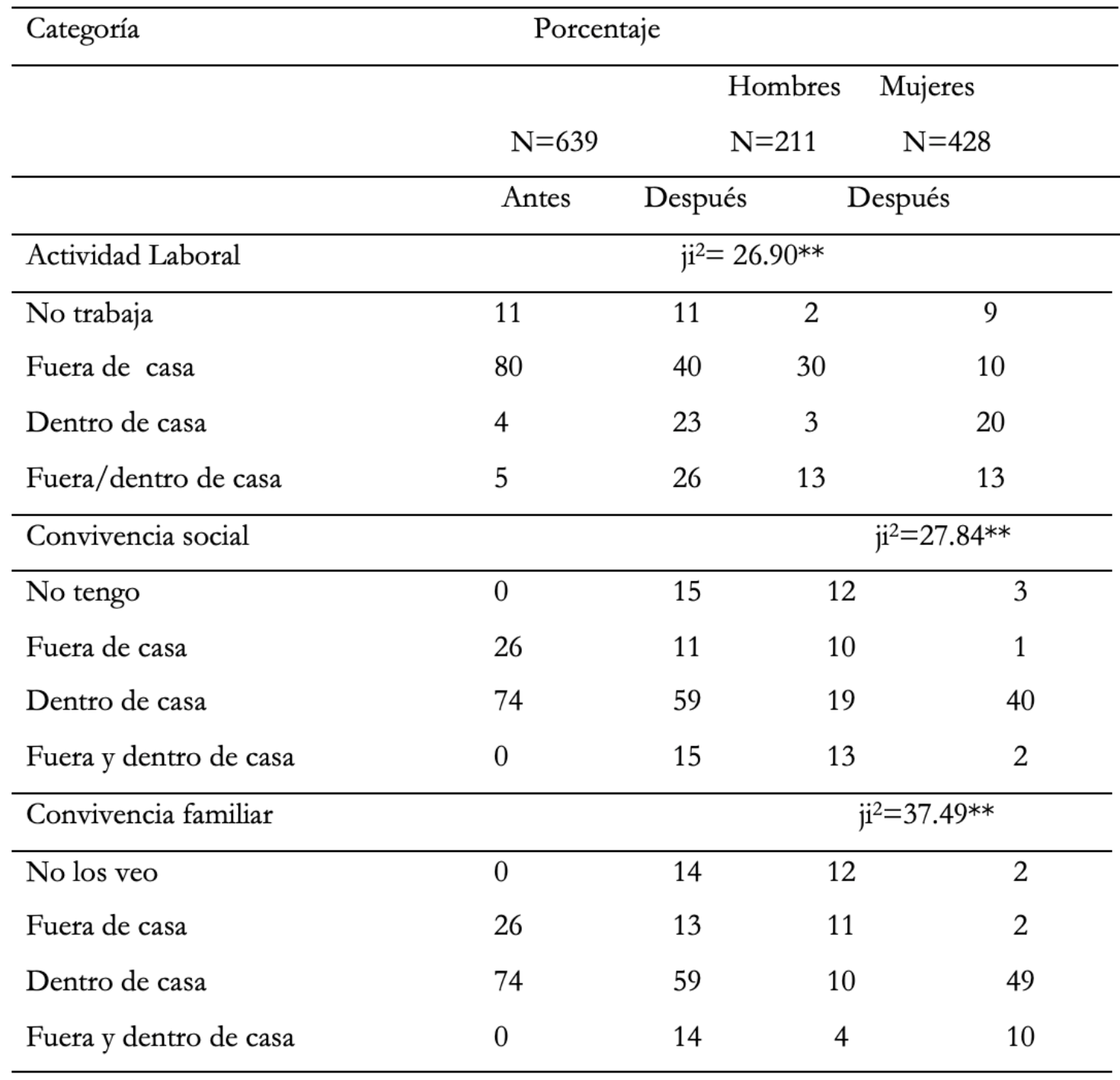

Nota. Elaboración propia $* * \mathrm{P}<0.0001$

Con relación al grado de aislamiento, los participantes informaron estar en casa sin salir en promedio 16.38 días (D.T.=6.8; rango=5-30), sin diferencias de género. El 52\% afirmó salir por alimentos y medicinas, el $37 \%$ informó seguir saliendo con precauciones (a comprar y/o pagar) pues no saben hacerlo en línea o no confían en este medio), el resto (11\%) afirmó no salir. 


\section{Violencia}

En cuanto a la violencia ( Tabla 3), se perciben comportamientos indicados por el violentómetro como alerta moderada en más de la mitad de la población (52\%), mientras que el $48 \%$ restante dejan pasar tiempo para que se pase el enojo. No se encontraron diferencias de género.

\section{Tabla 3}

\section{Tabla 3}

Tipos de violencia presentados durante el distanciamiento

\begin{tabular}{|c|c|}
\hline Tipo de violencia & Porcentaje \\
\hline Dar tiempo a que pase el enojo & 48 \\
\hline Culpamos & 7 \\
\hline Descalificamos & 8 \\
\hline Bromas hirientes & 15 \\
\hline Caricias agresivas & 14 \\
\hline Ignoramos o dejarnos de hablar & 8 \\
\hline
\end{tabular}

\section{Manejo gubernamental}

Sobre el manejo gubernamental de la pandemia (Tabla 4), se puede dividirse en positivo, donde el mayor porcentaje se encuentra en la efectividad (68\%), y con menor porcentaje califican las medidas como acertadas y pertinentes; en cuanto a los aspectos negativos en el manejo del gobierno, la mayoría lo consideró como confuso (61\%) y en menor porcentaje exagerado e improvisado. No se encontraron diferencias de género. 
Tabla 4

Tabla 4

Percepción del manejo gubernamental del COVID-19

\begin{tabular}{lclc}
\hline Categoría & $\begin{array}{c}\text { Percepción } \\
\%\end{array}$ & Categoría & Percepción \% \\
\hline Positiva & \multicolumn{3}{c}{$\mathrm{N}=639$} \\
\hline Efectivas & Negativa & \\
Acertadas & 37 & Exageradas & 35 \\
Pertinentes & 39 & Improvisadas & 61 \\
\hline
\end{tabular}

Nota. Elaboración propia

\section{Medio elegido de información}

El $47 \%$ se informan mediante internet, la T.V. y la radio (reportan escuchar las conferencias del gobierno). El 28\% se informa por internet (noticias o redes sociales), y el $25 \%$ sólo por radio o T.V. No se encontraron diferencias de género.

\section{Variables de salud general}

\section{Adherencia a conductas de prevención}

Al indagar sobre la práctica de medidas de prevención (Tabla 5), se tuvo que el $70 \%$ se lava las manos y el $45 \%$ usa el cubrebocas. Llama la atención que cuando se preguntó sobre la práctica de estas medidas en personas de su entorno inmediato, perciben que las realizan en menor medida que ellos. Se pudo observar que las personas no identifican el distanciamiento social como medida de prevención. Al parecer las personas entienden la función del distanciamiento, pero tienen dificultad para recordarlo y llevarlo a la práctica como una medida preventiva. Ellos consideran que $7 \%$ de su entorno practican la distancia social más que ellos (sobre todo jóvenes y menores, quienes les recuerdan que vean las marcas puestas en el piso). Lo cual marca la influencia entre las personas y su entorno en la creación y el establecimiento de normas subjetivas. No se encontraron diferencias de género en la percepción del entorno, no así, en la adherencia de los participantes, pues las mujeres se adhieren mejor (Tabla 5). 
Tabla 5

Tabla 5

Porcentaje de conductas de prevención realizadas por los participantes y su entorno inmediato en la muestra general y por género

\begin{tabular}{lllll}
\hline Categoría & \multicolumn{3}{c}{ Porcentaje } \\
\hline & Participante & Entorno & Participante & \\
\hline Adherencia a & & & Hombres & Mujeres \\
\hline Cubre bocas & 45 & 40 & $\mathrm{ji}^{2}=8.92^{*}$ & \\
Gel alcohol & 26 & 15 & 9 & 36 \\
Lavado manos & 70 & 70 & 20 & 18 \\
Careta acrílico & 10 & 8 & 2 & 80 \\
Distancia1.5mts & 39 & 46 & 19 & 20 \\
\hline
\end{tabular}

Nota. Elaboración propia $* \mathrm{P}<0.05$

En relación a la vulnerabilidad al contagio, se identificó que los varones (95\%), niños y adolescentes (100\%) tendieron a mostrar un sesgo positivo u optimista, afirmando "No, no me contagiaré". Mientras que las mujeres $\mathrm{y}$ adultos mayores tendieron a un sesgo pesimista, argumentando "me contagiaré porque tengo factores de riesgo, porque debo salir a trabajar, a hacer compras y trámites".

Conocimiento de medios de transmisión y medidas de protección

En cuanto al conocimiento sobre COVID-19 (Tabla 6), la mayoría de los participantes saben que es una enfermedad transmitida por virus (49\%), que la vía de acceso al cuerpo es por mucosas (ojos, nariz y boca) (68\%). Asimismo, identifican que el virus puede ser eliminado con jabón (73\%) o cloro (89\%), y comprenden que el riesgo de contagio se disminuye con distanciamiento social (86\%). Respecto a los factores de riesgo más identificados por los participantes se encuentran la diabetes (94\%) y la obesidad (95\%); otros factores menos identificados fueron ser varón y estar embarazada. No se encontraron diferencias de género (Tabla 6). 
Tabla 6

\section{Tabla 6}

Porcentaje sobre conocimientos y factores de riesgo ante al COVID-19

$$
\mathrm{N}=639
$$

Conocimientos sobre el COVID-19

$\% \quad$ Factores de riesgo

Es una enfermedad viral

No se contagia por la piel

Se elimina con jabón

Se elimina con cloro

Se evita con distancia social

No lo transmiten las mascotas

$\begin{array}{lll}49 & \text { Ser varón } & 65 \\ 68 & \text { Embarazo } & 60 \\ 73 & \text { Diabetes } & 94 \\ 89 & \text { Obesidad } & 95\end{array}$

86

49

Nota. Elaboración propia

\section{Impacto en la salud fisica en general}

Sobre el impacto en la salud física, en general $90 \%$ de la muestra percibe que se sentía bien y muy bien antes de la pandemia, ahora durante el distanciamiento social disminuyó al 80\%. En cuanto al porcentaje de personas que consideraron antes de la pandemia su salud regular, mala o muy mala, aumentó del $10 \%$ al $20 \%$. No se identificaron diferencias significativas de género.

\section{Impacto en la salud psicológica}

Sobre el estado emocional (Tabla 7), se pudo observar que los participantes presentaron cambios a raíz de la pandemia. Se destaca que el 37\% que consideraba su estado de ánimo muy bueno antes de la pandemia, durante el distanciamiento social bajó a $0 \%$. Por el contrario, los porcentajes de los que lo consideraban regular, malo o muy malo aumentaron del $6 \%$ al $37 \%$. En lo que respecta a la ansiedad, los que tenían un nivel leve y moderado sólo aumentaron 4 y 6 puntos porcentuales comparando su nivel antes y después de la pandemia. Contrario a esto, las personas con ansiedad severa antes de la pandemia, disminuyeron $10 \%$; lo cual puede atribuirse a los comentarios externados durante las aplicaciones. Ellos coincidían que ante la inseguridad en las calles, era tranquilizante tener a toda la familia en casa. En cuanto a la depresión, se observa un aumento en la presencia de ésta, ya que del $29 \%$ que se 
encontraba sin síntomas depresivos antes de la pandemia, sólo el $2 \%$ se mantuvo sin síntomas, por otro lado, se enfatiza el incremento en $18 \%$ de personas con sintomatología severa. En relación a las diferencias de género se observan diferencias significativas con mayor afectación en las mujeres (Tabla 7).

Tabla 7

\section{Tabla 7}

Impacto en la salud psicológica generado por el distanciamiento social en la muestra general y por género

\begin{tabular}{|c|c|c|c|c|}
\hline & Antes & Después & Después & \\
\hline \multirow[t]{2}{*}{ Grado de afectación } & \multicolumn{2}{|c|}{$\mathrm{N}=639$} & Hombres & Mujeres \\
\hline & \multicolumn{2}{|c|}{ Porcentaje } & $\mathrm{N}=211$ & $\mathrm{~N}=428$ \\
\hline Estado de ánimo & \multicolumn{4}{|c|}{$\mathrm{ji}^{2}=17.36^{* *}$} \\
\hline Muy bueno & 37 & 0 & 0 & 0 \\
\hline Bueno & 55 & 53 & 47 & 6 \\
\hline Regular & 3 & 29 & 20 & 9 \\
\hline Malo & 3 & 3 & 1 & 2 \\
\hline Muy malo & 0 & 5 & 1 & 4 \\
\hline Ansiedad & \multicolumn{4}{|c|}{$\mathrm{ji}^{2}=19.98^{* *}$} \\
\hline Leve & 26 & 30 & 19 & 11 \\
\hline Moderada & 48 & 54 & 7 & 37 \\
\hline Severa & 26 & 16 & 3 & 13 \\
\hline Depresión & \multicolumn{4}{|c|}{$\mathrm{ji}^{2}=34.65^{* *}$} \\
\hline Sin depresión & 29 & 2 & 1 & 1 \\
\hline Leve & 39 & 45 & 39 & 6 \\
\hline Moderada & 30 & 33 & 18 & 15 \\
\hline Severa & 2 & 20 & 2 & 18 \\
\hline
\end{tabular}

Nota. Elaboración propia $* * \mathrm{P}<0.0001$

\section{Cambios conductuales}

Al evaluar el estilo de vida, se observó que 49\% tienen dificultad para comer porciones normales de comida, aumentando el tamaño y la frecuencia con la que consumen alimentos. El 29\% tiene trastornos del sueño ( $15 \%$ problemas para despertar y $14 \%$ para dormir). El $17 \%$ 
tiene problemas para establecer horarios de sueño. Con relación a los comportamientos repetitivos más frecuentes $74 \%$ de los participantes tiene la necesidad de estar frente a alguna pantalla. El 50\% siente deseos de dormir todo el tiempo. El 22\% informó tener deseos de estar activo, en compañía de alguien todo el tiempo y controlando lo que otros hacen; así mismo, entre el 10\% y $13 \%$ dicen tener necesidad de quejarse, informarse, negar/minimizar lo que ocurre o comprar víveres, el 6\% no presentó ninguna y sólo $1 \%$ dijo presentar todas. No se encontraron diferencias de género.

\section{Adicciones}

Se observó que el $24 \%$ no muestra ninguna conducta adictiva, el $45 \%$ dice consumir más productos azucarados, el $8 \%$ aumentó su consumo de tabaco, $19 \%$ el consumo de alcohol y $4 \%$ informa presentar todas las anteriores. Tampoco se encontraron diferencias de género.

\section{Discusión}

A partir de los resultados obtenidos, en las variables sociodemográficas, se tiene que la mayoría de los participantes son adultos, mujeres, pertenecientes a familias nucleares y extensas (durante la encuesta refirieron bienestar general al decidir reagruparse para minimizar gastos). Esto redunda en menor preocupación financiera, muestra cierto factor protector, así como la adaptabilidad y cohesión de las familias participantes, ya que además, dos terceras partes logran encontrar alguna forma de trabajar desde o fuera de casa, aludiendo a la redistribución de roles y tareas en el hogar lo que hace diferir esta muestra de las de otros estudios (Lai et al., 2020; Li, Yang et al., 2020; Liu et al., 2020; McMullan et al., 2016). Sobre la variable clase social recogida mediante el índice Hollingshead (1975), los dos indicadores son elevados, por lo que se considera que hubo cierto sesgo, respecto al grueso de la población de la UMF, lo que se retomará más adelante.

$\mathrm{Al}$ analizar los aspectos del impacto de la pandemia en su vida laboral un tercio se ve afectado, pues aun cuando son profesionistas o microempresarios, se ven forzados a cerrar por no pertenecer al ramo de los comercios o servicios esenciales.

Llama la atención que no hay diferencias de género antes ni después de la pandemia, lo que al parecer puede relacionarse con la flexibilidad de roles en las familias que puede fungir como un factor protector. En cuanto a los ámbitos social y familiar se observa un patrón similar, es decir, ambas se reducen a la mitad y sólo un poco menos de la quinta parte definitivamente dejan de tener actividades sociales y familiares. Al comparar por género si se notan diferencias, los hombres socializan más al exterior y las mujeres conviven más con la familia dentro de casa.

Cuando se exploró el grado de confinamiento, la población informó que no logra mantenerse en casa, por buscar ingresos o por gestionar 
compras y pagos (aun no dominan suficientemente el uso de medios digitales). Con lo cual, también se ve uno de los más difundidos efectos de la pandemia, que implica la necesidad de una migración de los modos presenciales a distancia en todas las esferas sociales. En la evaluación del impacto conductual, como lo indican Jacques et al. (2019), Hawryluck et al. (2004) y Jeong et al. (2016), se observó una importante afectación en los hábitos alimenticios y de sueño. También informan que con mucha frecuencia quieren consumir alimentos con azúcar (Rada et al., 2005). Respecto a los hábitos de sueño se encuentran dificultades para dormir y despertar, lo que pudiera relacionarse con estados de ansiedad y el sedentarismo que ha generado la pandemia (Lin et al., 2021). En relación a las diferencias de género se coincide con los autores que informan trastornos de conducta similares en hombres y mujeres (Terán-Pérez et al., 2021; Yuksel et al., 2021). Hecho preocupante para la población de adultos en México, la cual es más vulnerable por presentar sobrepeso, obesidad y diabetes (INEGI \& INSP, 2018). Otro dato importante es que se da la presencia de comportamientos repetitivos. Entre los más frecuentes están los deseos de estar frente a pantallas (Teléfonos móviles, tabletas, computadoras, video juegos, T.V.) y querer dormir todo el tiempo, lo cual concuerda con el impacto en los hábitos de sueño, como informa Robles et al. (2020).

$\mathrm{Al}$ evaluar la presencia de violencia y adicciones dada la vulnerabilidad e incertidumbre que genera la pandemia (Díaz et al., 2007; Jacques et al., 2019; Rada et al., 2005; Sadati et al., 2020; Taylor et al., 2008), ambos fenómenos están presentes aunque se manifiestan moderadamente, ya que los participantes observan la necesidad de consumir productos azucarados y el consumo de alcohol, como los más frecuentes, sin que se encontraran diferencias de género. En lo que respecta a la violencia no se observa en grados severos, sin embargo, se identifica en más de la mitad de la muestra, lo cual indica la necesidad de capacitar en habilidades sociales y comunicación asertiva para prevenir que ésta crezca.

Respecto a la salud en general, se ve también una percepción de deterioro por trastornos de comportamiento, igual que en el caso de los síntomas depresivos, los cuales en esta muestra como en la literatura se presentan más en mujeres (Betancourt-Ocampo et al., 2021; HuarcayaVictoria, 2020; Robles et al., 2020; Saldívar-Garduño \& Ramírez-Gómez, 2020; Terán-Pérez et al., 2021; Zaccarelli et al., 2006) notándose que las personas que no tenían síntomas depresivos empiezan a tenerlos y los que ya los tenían se agravan. Lo que no ocurre con la ansiedad, pues los participantes informan estar "más tranquilos" al estar todos en casa por la inseguridad que se vive en las calles.

Al evaluar el conocimiento sobre la nueva enfermedad COVID-19 y los factores de riesgo, los resultados fueron positivos un elevado porcentaje de los participantes tiene conocimientos satisfactorios. No obstante al evaluar las conductas preventivas que realizan, a pesar de conocer todas, sólo practican el uso de cubrebocas, alcohol-gel y lavado de manos, el resto de medidas son poco practicadas, incluso la distancia social, no fue identificada como un "medio de prevención". 
Sobre los medios para informarse, casi la mitad lo hace mediante internet, T. V. o radio. Por otro lado, al valorar el desempeño del gobierno, para considerar el grado de control delegado por los participantes, se corrobora lo expuesto por Caldera-Villalobos et al. (2020) pues sobresalen calificativos como confuso, exagerado e improvisado, lo cual puede estar restando credibilidad a las indicaciones. Así mismo, entre las percepciones positivas, más de dos terceras partes cree que las medidas han sido efectivas, argumentando que ellos no se han contagiado ni personas cercanas. Este dato subraya la relevancia de la identificación de los factores vinculados a los sesgos en la percepción de la pandemia ( $\mathrm{Li}$, Wang et al., 2020).

Respecto a los motivos de contagio, como se indicó, entre los varones y adolescentes se encontró un sesgo positivo, considerando que no se contagiarán. En las mujeres y adultos mayores se notó un sesgo negativo, aludiendo a los factores de riesgo que presentan, para contagiarse, lo que puede ser muestra de mayor estrés como informó Betancourt-Ocampo et al. (2021). Sin embargo, ninguno de los grupos mencionados considera la práctica frecuente y correcta de conductas de prevención para como un factor protector. Un hallazgo importante fue el observar cómo las normas subjetivas modelan el comportamiento como lo indican Cho y Lee (2015), pues las personas y su entorno inmediato mostraron un comportamiento muy similar en el empleo de medidas preventivas, notando que el distanciamiento social, sigue representando reto (sobre todo en los de mayor edad), por olvido o por rechazo social, pues se sienten forzados al contacto físico. Este último hallazgo, puede ser extrapolable a diferente comunidades de latinos donde debe modificarse la idea de que negarse al contacto físico por salud es sinónimo de descortesía.

Así mismo, empezar a ver este modelo de investigación e intervención como útil en la nueva normalidad es una de las contribuciones de este estudio, pues constituye la primera evidencia que se tiene del empleo de plataformas y alfabetización digital en esta comunidad. El trabajo a distancia en la UMF, optimiza recursos materiales y humanos, ya que como se vio a lo largo del estudio: a) El levantamiento de datos a distancia, en modo de entrevista colectiva fue exitoso y con adecuado rigor metodológico; b) La estrategia de inclusión de los adultos acompañados por adolescentes de su familia, facilitó el escenario digital y la asistencia de todos los grupos etarios; y c) La participación mayoritaria de las mujeres en el estudio, pone de manifiesto la inequidad de género en relación a la salud, que alude al estereotipo "las mujeres son encargadas de la salud familiar". Sin embargo, se considera que esta nueva modalidad a distancia síncrona o asíncrona de atención a la salud, puede representar un punto hacia la equidad de género, fomentando el cuidado responsable de la salud de todas y todos, pues la atención a distancia les posibilitaría asistir y estar informados para cuidarse. 


\section{Limitaciones}

Aun cuando la estrategia para recoger los datos del estudio fue exitosa. Esto impuso un sesgo en la muestra, pues $9 \%$ de los convocados no asistieron, para quienes se ha tenido que recurrir a estrategias presenciales de atención. El sesgo respecto a nivel de estudios (bachillerato o licenciatura) y la ocupación (micro empresarios o profesionistas) en más de dos tercios de la muestra se puede ver a la luz del rezago y desigualdad en las oportunidades para el manejo digital óptimo en nuestro país; originado primero, por aspectos de tipo material (acceso a internet y a dispositivos digitales); y segundo, por desventajas educativas (alto índice de analfabetismo). Ambos factores que con estudios como este se visibilizan y ya están siendo atendidos en países en vías de desarrollo como el nuestro.

\section{Conclusiones}

Finalmente, aun cuando la muestra no presenta alta vulnerabilidad, requieren apoyo. Se debe considerar incidir desde los niveles más próximos a las comunidades desde las UMF en las medidas de alfabetización digital, prevención de riesgos y conocimiento de la enfermedad por COVID-19 (considerando las características de la población de acuerdo a los sesgos positivos/negativos y las normas subjetivas). De igual forma, debido a que la muestra presenta un deterioro en la salud en general y psicológica, es prioritario mantener intervenciones a distancia. Considerando los resultados obtenidos, se debe incidir en la equidad de género para el cuidado de la salud, incentivando a los varones a participar en su autocuidado y a las mujeres para que además de cuidar a otros cuiden de sí mismas. La inclusión de adolescentes debe mantenerse implementando la desprofesionalización de los grupos multidisciplinarios hacia la comunidad mediante migración de los programas preventivos de salud al modo no presencial. Este estudio ha permitido continuar en la nueva normalidad, manteniendo una comunidad más inclusiva vinculando generaciones mediante el uso de medios digitales.

\section{Agradecimientos}

A todos los participantes, especialmente a los adolescentes que apoyaron instruyendo digitalmente sus familias.

\section{Referencias}

Andersen, K. G., Rambaut, A., Lipkin, W. I., Holmes, E. C., \& Garry, R. F. (2020). The proximal origin of SARS-CoV-2. Nature Medicine, 26(4), 450-452. https://doi.org/10.1038/s41591-020-0820-9 
Aragón-Nogales, R., Vargas-Almanza, I., \& Miranda-Novales, M. G. (2019). COVID-19 por SARS-CoV-2: la nueva emergencia de salud. Revista Mexicana de Pediatría, 86(6), 213-218.

BBC News. (2020a). Coronavirus confirmed as pandemic by World Health Organization. BBC News. https://www.bbc.com/news/world-51839944

BBC News. (2020b). Coronavirus: Por qué covid-19 se llama así y cómo se nombran los virus y las enfermedades infecciosas. BBC News. https://ww w.bbc.com/mundo/noticias-51912089

Betancourt-Ocampo, D., Reyes-Zamorano, E., Romo-Parra, H., ToledoFernández, A., \& González-González, A. (2021). Psychological impact of sociodemographic factors and medical conditions in older adults during the COVID-19 pandemic in Mexico. Salud Mental, 43(6), 293-301. http s://doi.org/10.17711/SM.0185-3325.2020.040

Brooks, S. K., Webster, R. K., Smith, L. E., Woodland, L., Wessely, S., Greenberg, N., \& Rubin, G. J. (2020). The psychological impact of quarantine and how to reduce it: Rapid review of the evidence. The Lancet, 395(10227), 912-920. https://doi.org/10.1016/S0140-6736(20)304608

Caldera-Villalobos, C., Garza-Veloz, I., Martínez-Avila, N., Delgado-Enciso, I., Ortiz-Castro, Y., Cabral-Pacheco, G. A., \& Martinez-Fierro, M. L. (2020). The coronavirus disease (COVID-19) challenge in Mexico: A critical and forced reflection as individuals and society. Frontiers in Public Health, 8 , 337. https://doi.org/10.3389/fpubh.2020.00337

Centers for Disease Control and Prevention (CDC). (2020). Coronavirus: Human Coronavirus Types. CDC. https://www.cdc.gov/coronavirus/typ es.html

Cho, H. C., \& Lee, J. S. (2015). The influence of self - efficacy, subjective norms, and risk perception on behavioral intentions related to the H1N1 flu pandemic: A comparison between Korea and the US. Asian Journal of Social Psychology, 18(4), 311-24. https://onlinelibrary.wiley.com/doi/abs /10.1111/ajsp.12104

Del Barrio, V., \& Carrasco, M. A. (2004). CDI. Inventario de depresión infantil. TEA Ediciones.

Díaz, D., Abarca, A. B., Sutil, L., \& Shweiger, I. (2007). Argumento para una propuesta psicosocial del trauma II: el bienestar, patrón de salud y trastorno. En S. Yubero Jiménez, E. Larrañaga Rubio \& A. Blanco Abarca (Eds.), Convivir con la violencia: un análisis desde la psicología y la educación de la violencia en nuestra sociedad (pp. 47-70). Ediciones de la Universidad de Castilla-La Mancha.

El Instituto Nacional de Estadística y Geografía (INEGI) \& el Instituto Nacional de Salud Pública (INSP). (2018). Encuesta Nacional de Saludy Nutrición (ENSANUT). https://ensanut.insp.mx/encuestas/ensanut20 18/doctos/metodologia/ensanut_2018_diseno_conceptual.pdf

Galindo-Vázquez, O., Ramírez-Orozco, M., Costas-Muñiz, R., MendozaContreras, L. A., Calderillo-Ruíz, G., \& Meneses-García, A. (2020). Symptoms of anxiety and depression and self-care behaviors during the COVID-19 pandemic in the general population. Gaceta Médica de México, 156(4), 294-301. http://dx.doi.org/10.24875/gmm.m20000399

Gobierno de la Ciudad de México \& Secretaría de Salud. (2020). Todo sobre el COVID-19. https://coronavirus.gob.mx/ 
Gobierno de la Ciudad de México \& Secretaría de Salud. (2021). Servicios COVID-19 para la población. https://covid19.cdmx.gob.mx/

González, D. A., Reséndiz, R. A., \& Reyes, L. I. (2015). Adaptation of the BDIII in México. Salud Mental, 38(4), 237-244. https://doi.org/10.17711/s m.0185-3325.2015.033

Hawryluck, L., Gold, W. L., Robinson, S., Pogorski, S., Galea, S., \& Styra, R. (2004). SARS Control and psychological effects of quarantine, Toronto, Canada. Emerging Infectious Diseases, 10(7), 1206-1212. http://dx.doi.o $\mathrm{rg} / 10.3201 /$ eid1007.030703

Hollingshead, A. (1975). Four factor index of social status [Unpublished manuscript]. Yale University. http://fcon_1000.projects.nitrc.org/indi/e nhanced/assessments/ses-child.html

Huarcaya-Victoria, J. (2020). Consideraciones sobre la salud mental en la pandemia de COVID-19. Revista Peruana de Medicina Experimental y Salud Pública, 37(2), 327-334. https://doi.org/10.17843/rpmesp.2020.3 72.5419

Instituto Politécnico Nacional (IPN). (2009). Violentómetro. Portal del Instituto Politécnico Nacional. https://www.ipn.mx/

Inter-Agency Standing Committee (IASC). (2007). OMS | Guia del IASC sobre salud mental y apoyo psicosocial en emergencias humanitarias y catástrofes. World Health Organization. http://www.who.int/hac/techguidance/ias c-poster/es/

Jacques, A., Chaaya, N., Beecher, K., Ali, S. A., Belmer, A., \& Bartlett, S. (2019). The impact of sugar consumption on stress driven, emotional and addictive behaviors. Neuroscience and Biobehavioral Reviews, 103, 178199. https://doi.org/10.1016/j.neubiorev.2019.05.021

Jeong, H., Yim, H. W., Song, Y.-J., Ki, M., Min, J.-A., Cho, J., \& Chae, J.H. (2016). Mental health status of people isolated due to Middle East Respiratory Syndrome. Epidemiology and Health, 38, e2016048. https:// doi.org/10.4178/epih.e2016048

Lai, J., Ma, S., \& Wang, Y. (2020). Factors associated with mental health outcomes among health care workers exposed to coronavirus disease 2019. Journal of The American Medical Association, 3(3), e203976. https://doi: 10.1001/jamanetworkopen.2020.3976

Landa-Blanco, M., Mejía, C. J., Landa-Blanco, A. L., Martínez-Martínez, C. A., Vásquez, D., Vásquez, G., ... \& Montoya, B. D. (2021). Coronavirus awareness, confinement stress, and mental health: Evidence from Honduras, Chile, Costa Rica, Mexico and Spain. Social Science \& Medicine, 277, 113933. https://doi.org/10.1016/j.socscimed.2021.1139 33

Li, S., Wang, Y., Xue, J., Zhao, N., \& Zhu, T. (2020). The impact of COVID-19 epidemic declaration on psychological consequences: A study on active weibo users. InternationalJournal of Environmental Research and Public Health, 17(6), 2032. https://doi.org/10.3390/ijerph17062032

Li, W., Yang, Y., Liu, Z. H., Zhao, Y. J., Zhang, Q., Zhang, L., ... \& Xiang, Y. T. (2020). Progression of mental health services during the COVID-19 outbreak in China. International Journal of Biological Sciences, 16(10), 1732-1738. https://doi.org/10.7150/ijbs. 45120

Lin, Y., Liu, S., Li, S., Zuo, H., \& Zhang, B. (2021). Relationships between changes in sleep patterns and sleep quality among Chinese during the 2019 
coronavirus disease outbreak. Sleep Medicine. Advance online publication. https://doi.org/10.1016/j.sleep.2021.01.021

Liu, S., Yang, L., Zhang, C., Xiang, Y., Liu, Z., Hu, S. (2020). Online mental health services in China during the COVID-19 outbreak. The Lancet Psychiatry, 7(4), e17-e18. https://doi.org/10.1016/S2215-0366(20)3007 7-8

McMullan, C., Brown, G. D., \& O'Sullivan, D. (2016). Preparing to respond: Irish nurses' perceptions of preparedness for an influenza pandemic. International Emergency Nursing, 26, 3-7. https://doi.org/10.1016/j.ienj .2015 .10 .004

Organización Mundial de la Salud (OMS). (2020). Brote de enfermedad por coronavirus (COVID-19). Nuevo coronavirus 2019. https://www.who.in t/es/emergencies/diseases/novel-coronavirus-2019

Organización Panamericana de la Salud (OPS). (2010). PAHO/WHO Health Emergencies-Apoyo psicosocial en emergenciasy desastres $\mid$ OPS/OMS. Pan American Health Organization/ World Health Organization. https://w ww.paho.org/disasters/index.php?option $=$ com_content\&view $=$ article \&id=1362:psychosocial-support-in-emergencies-and-disasters\&Itemid= 924\&lang=es

Organización Panamericana de la Salud (OPS). (2012). PAHO/WHO Health Emergencies-Mental Health and Psychosocial Support in Disaster Situations in the Caribbean | OPS/OMS. Pan American Health Organization / World Health Organization. https://www.paho.org/disas ters/index.php?option=com_content\&view $=$ article\&id $=1649$ : mental $-\mathrm{h}$ ealth-and-psychosocial-support-in-disaster-situations-in-the-caribbean $\&$ Itemid $=807 \&$ lang $=$ es

Organización Panamericana de la Salud (OPS). (2016). Guía técnica de salud mental en situaciones de desastresy emergencias-OPS/OMS $\mid$ Organización Panamericana de la Salud. https://www.paho.org/es/documentos/guia-t ecnica-salud-mental-situaciones-desastres-emergencias

Rada, P., Avena, N. M., \& Hoebel, B. G. (2005). “Adicción” al azúcar: ¿̇mito o realidad? Revista Venezolana de Endocrinología y Metabolismo, 3(2),02-12.

Rajkumar, R. P. (2020). COVID-19 and mental health: A review of the existing literature. Asian Journal of Psychiatry, 52, 102066. https://doi.org/10.10 16/j.ajp.2020.102066

Ramírez, L. P. G., Arriaga, R. J. M., Hernández-Gonzalez, M. A., \& De la RocaChiapas, J. M. (2020). Psychological distress and signs of post-traumatic stress in response to the COVID-19 health emergency in a Mexican sample. Psychology Research and Behavior Management, 13, 589-597. http s://doi.org/10.2147/PRBM.S259563

Robles, R., Rodríguez, E., Vega-Ramírez, H., Álvarez-Icaza, D., Madrigal, E., Durand, S., \& Morales-Chainé S. (2020). Mental health problems among healthcare workers involved with the COVID-19 outbreak. Brazilian Journal of Psychiatry. https://doi.org/10.1590/1516-4446-2020-1346

Robles, R., Varela, R., Jurado, S., \& Páez, F. (2001). Versión mexicana del Inventario de Ansiedad de Beck: propiedades psicométricas. Revista Mexicana de Psicología, 18(2), 211-218.

Sadati, A. K., B Lankarani, M. H., \& Bagheri Lankarani, K. (2020). Risk society, global vulnerability and fragile resilience; sociological view on the 
coronavirus outbreak. Shiraz E-Medical Journal, 21(4), e102263. https:/ /doi.org/10.5812/semj.102263

Saldívar-Garduño, A., \& Ramírez-Gómez, K. E. (2020). Salud mental, género y enseñanza remota durante el confinamiento por el COVID-19 en México. Persona: Revista de la Facultad de Psicologia, (23), 11-40. https://doi.org/ 10.26439/persona2020.n023(2).5011

Secretaria de Salud. (2020). Segundo Informe de labores de Salud 2019-2020. https://www.gob.mx/salud/documentos/segundo-informede-labores-ssa-2019-2020

Taylor, M. R., Agho, K. E., Stevens, G. J., \& Raphael, B. (2008). Factors influencing psychological distress during a disease epidemic: Data from Australia's first outbreak of equine influenza. BMC Public Health, 8, 347. https://doi.org/10.1186/1471-2458-8-347

Terán-Pérez, G., Portillo-Vásquez, A., Arana-Lechuga, Y., Sánchez-Escandón, O., Mercadillo-Caballero, R., González-Robles, R. O., \& VelázquezMoctezuma, J. (2021). Sleep and mental health disturbances due to social isolation during the COVID-19 Pandemic in Mexico. International Journal of Environmental Research and Public Health, 18(6), 2804. https ://doi.org/10.3390/ijerph18062804

Tesini, B. L. (2020). Coronavirus and acute respiratory syndromes (COVID-19, MERS and SARS)_Infections. ManualMSD version for generalpublic. ht tps://www.msdmanuals.com/es/hogar/infecciones/virus-respiratorios/c oronavirus-y-s\%C3\%ADndromes-respiratorios-agudos-covid-19-mers-ysars

Valdez, L. C., Gutiérrez, M. L. E., \& Baena, V. G. (2020). Variables predictoras de las respuestas psicológicas ante Covid-19: un estudio en México. Ciencia y Sociedad, 45(3), 7-23. https://doi.org/10.22206/cys.2020.v45i 3.pp7-23

World Health Organization (WHO). (2004). Invertir en salud mental. Organización Mundial de la Salud. https://apps.who.int/iris/handle/106 $65 / 42897$

Yuksel, D., McKee, G. B., Perrin, P. B., Alzueta, E., Caffarra, S., Ramos-Usuga, D., \& Baker, F. C. (2021). Sleeping when the world locks down: Correlates of sleep health during the COVID-19 pandemic across 59 countries. Sleep Health, 7(2), 134-142. https://doi.org/10.1016/j.sleh.2020.12.008

Zaccarelli, M., Rodríguez, J., Pérez, R., \& Organización Panamericana de la Salud. (2006). Guía práctica de salud mental en situaciones de desastres. Organización Panamericana de la Salud, Oficina Regional de la Organización Mundial de la Salud. 